\title{
Improving the Energy Efficiency in Mobile Ad-Hoc Network Using Learning-Based Routing
}

\author{
Vijay Vasanth Aroulanandam ${ }^{1}$, Thamarai Pugazhendhi Latchoumi², Karnan Balamurugan ${ }^{3 *}$, \\ Tiruchengode Lakshmanasamy Yookesh ${ }^{4}$ \\ ${ }^{1}$ Department of Computer Science and Engineering, Vel Tech Rangarajan Dr. Sagunthala R\&D Institute of Science and \\ Technology, Chennai 600062, India \\ ${ }^{2}$ Department of Computer Science and Engineering, VFSTR (Deemed to be University), AP 522213, India \\ ${ }^{3}$ Department of Mechanical Engineering, VFSTR (Deemed to be University), AP 522213, India \\ ${ }^{4}$ Division of Mathematics, Department of Science and Humanities, VFSTR (Deemed to be University), AP 522213, India
}

Corresponding Author Email: adc3_iqac@vignan.ac.in

https://doi.org/10.18280/ria.340312

Received: 15 February 2020

Accepted: 2 May 2020

\section{Keywords:}

learning-based routing, neural networks, node range adjustment, sequential learning, weighted clustering

\begin{abstract}
Improving Mobile Ad-Hoc Network (MANET) performance is a tedious task because of the dynamic and uncertain characteristics of the nodes. MANET nodes connected to multiple applications that involve a high exchange of data. To add reliability to the application services, MANETs optimized through dedicated load balancing schemes. The Dynamic Range Clustering (DRC) algorithm associated with Learning-based Routing (LR) used in the present work to improve the energy efficiency and the nature of the network's data handling. DRC design focuses on selecting the head of the cluster and maintaining stability over the cluster. LR selects unique neighbors that assist in ensuring the efficient, non-congested communication of data exchange. An effort has made to combine the two incompatible methods to increase the performance of the network over differential network traffic. The proposed approach tested using simulations and measures output by comparative analysis.
\end{abstract}

\section{INTRODUCTION}

Within a MANET, the most common wireless media interlocks the self-disciplined mobile host groups for communication. The host nodes separated by the physical distance to which the multi-hop connections used. This network designed for unique applications that don't depend on any backbone infrastructure [1]. With MANETs rapid development technology, it employed industrial, residential, military, and science applications in real-time. The positive position of this network was operating with fewer resources in an emergency scenario would also serve as a temporary backup. The nodes/mobile hosts are dynamic and move around freely on encompassing the area that permits the network to cover the entire region. As mentioned earlier, the nodes rely on their neighbors for multi-hop communications over the spread region.

With the mobility feature, the nodes can maintain and restructure of the network topology [2]. Communication involves data transmission between one-hop and multi-hop neighbors that was accomplished using routing and transmission protocol. The nodes, which encompass a wide region, are dependent on their neighbors for data transmission and receiving [3]. The confronted MANETs weakness was the absence of a central controller and its autonomous character. They have a significant effect on the daily process of node contact and networking. Scaling the nodes in the network was also tedious because of built-in mobility which causes repeated connection breakage and data loss [4].

The systematic clustering methodology used to address the various issues related to data transmission and scalability. As per the call of the researcher, clustering helps in network stability through correlating links and communication through a hierarchy. The encompassing nodes are segregated as small enclosures called clusters, each attempt to hold two different node types [5]. The Cluster Head $(\mathrm{CH})$ and the Cluster Member (CM) are the two categories of nodes in the interconnected groups/enclosures. $\mathrm{CHs}$ are responsible for communication, scheduling, medium allocation, and aggregation for the maintenance of network endurance [6]. The size of the cluster was always different and depends on the number of neighbors who make requests. The range of communication of the cluster depends on the radio range of the $\mathrm{CH}$. The battery power of the nodes was maintained in a less density cluster, while the cluster energy was exhausted at an early stage in the case of a high-density cluster [7]. The dynamic nature of the network, the excessive exchange of information, and the stability of the network has been controlled by the cluster head with better resource utilization. In other words, clustering improves network scalability, resource utilization, and seamless connectivity despite network coverage. Clusters distribute tasks across the network to prevent overloading at the node level and to harmonize communications. Routing endurance and path set up in a clustering environment minimizes the impact of bottlenecks to achieve optimal network performance [8].

To achieve stability, unpredictable network traffic communicates between single and multi-track nodes increased network traffic requires a formal load balancing scheme to effectively distribute incoming data between available routes [9]. Load balancing was intended to enhance 
the use of resources by the network controlling exploitation. Multi-path communication improves communication rates with increased bandwidth accessibility [10]. Effective load balancing focuses on estimating and disseminating incoming traffic.

In the load balancing process, workloads were distributed across the available nodes for overloading and resource exploitation [11]. Additive concern was the selection of appropriate nodes to ensure reliable forward-capacity data, avoiding packet loss and replication. Also, nodes must ensure proper route paths to the destination for the delivery of the received data [12]. Lack of exhaust network bandwidth, node capacity, device storage, or inadequate load balancing produces node level traffic imbalance that causes storage overload that will result in packet loss and increased delay [13]. A fair play load balancing method with a proper node selection algorithm in the network improves the nature of load handling and minimizes the exploitation of resources between nodes.

\section{RELATED WORKS}

In wireless sensor networks, a node placement method was proposed for load balancing. By restricting incoming network traffic, this approach allows minimal-hop data transmission. In comparison, this node placement strategy was costeffective and energy-efficient, and it fails to represent the number of control messages exchanged [14].

Delay aware load balancing technique was developed to reduce the wireless network adjournment and packet segregation errors. Through analyzing the traffic and packet flow to evaluate the sequence of the packet to be transmitted, this algorithm best suited for multi-path communication [15].

The authors proposed a clustering scheme with a selforganizing attribute to ensure scalability and make the network stable. This clustering was inspired by birds flocking behavior to dynamically adjust cluster size to minimize congestion. This clustering works best in Group mobilityinduced MANETs [16]. They ended up with a result that guaranteed node durability and robustness with lower energy requirements.

The load balancing would predict the optimal route by using Ant Colony Optimization (ACO) to find appropriate pathways. The path preferences were determined using metrics such as delay, bandwidth, and accessibility to the neighbor. ACO technique has become an effective method to enhance network performance, and it does not limit the exchange of control messages when the number of paths increases [17].

A generic clustering algorithm [18] was indeed designed to improve the stability of group-based networks and the cluster lifetime. The algorithm developed was based on the density of the nodes and the alliance between them. The writers in $[19,20]$ suggest a central controller that had already implemented semi-matching load balancing for WLAN. To spread the uneven network loads, the central controller acquires knowledge of the active channel time. The matching process was performed using the Kuhn-Munkres (K-M) algorithm to maximize network throughput and this method was least reliable for multi-rate WLANs problems [21].

\section{PROPOSED METHOD}

\subsection{Network model}

In a distributed $\mathrm{X}^{*} \mathrm{Y}$ dimension network, the mobile nodes are defined by $\{\mathrm{n}(1,) \mathrm{n}(2)$, etc. The nodes share the information among themselves using wireless links (V). If the network was represented as a graph $G$ then, $G=(N, V)$ would define node sets and links.

A node ' $i$ ' has a wireless link with its neighbor ' $j$ ' if dist ( $i$, j) < R (i)(j), then R is the ratio within the I or j range.

The nodes in the network are grouped into clusters whereby the $\mathrm{CH}$, pursued by its $\mathrm{CM}$, guided every cluster. The $\mathrm{CH}$ aggregates its $\mathrm{CM}$ data and ensures distribution at the Base Station (BS). Figure 1 shows a sample cluster network.

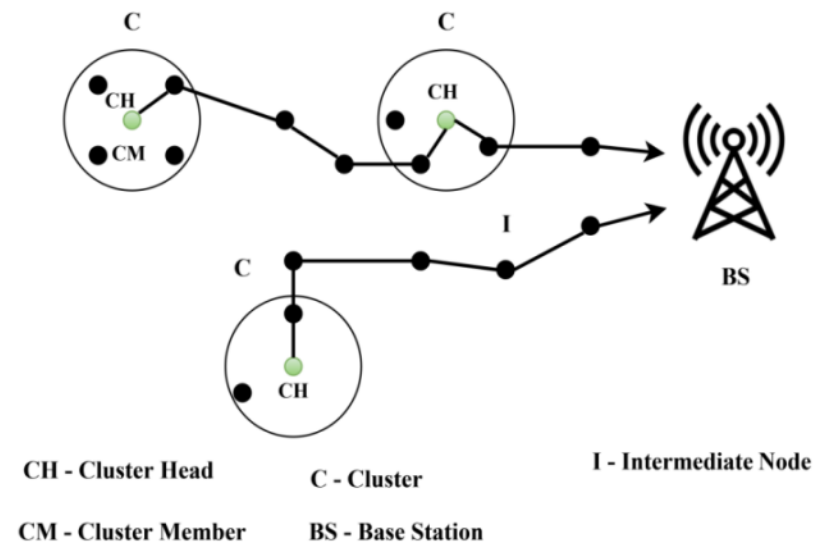

Figure 1. The proposed cluster network model

\subsubsection{Assumptions}

(1) Intermediate nodes are not part of any cluster but a member of $\mathrm{X}^{*} \mathrm{Y}$.

(2) If the $\mathrm{CH}$ is present in one-hop, it communicates directly with the BS; otherwise, it relies on its neighbors for contact.

(3) The initial $\mathrm{CH}$ is the first communicating node, and the later $\mathrm{CH}$ is chosen based on appropriate constraints.

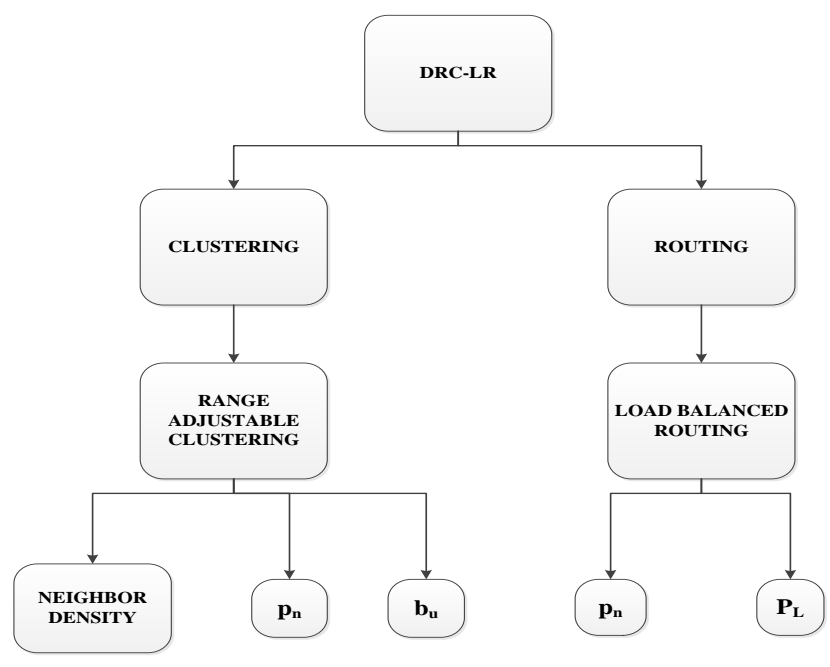

Figure 2. DRC-LR process 


\subsection{Dynamic range clustering (DRC) with learning-based routing (LR) scheme}

The proposed DRC-LR is to increase the ability of MANET nodes to handle the load. Figure 2 illustrates the overall DRC-LR process.

\subsection{Dynamic range clustering (DRC) algorithm}

DRC makes a collection of $\mathrm{CH}$ simpler across a set of learning processes. A neural network evaluation for the option of specific $\mathrm{CH}$ affects the learning process. Besides, the learning process determines the number of optimum $\mathrm{CM}$ required to relay the current network traffic.

Two explicit and hidden constraints are executed for each of the learning iterations performed by neural network evaluation. The two explicit constraints are assessed using neighboring density and use of bandwidth. For several packets, the implicit constraint decides the range of the current $\mathrm{CH}$. Previously, the head of the cluster is chosen using the variable weight function $(\nabla w)$ derived from the neural computation output.

The weight function to pick a $\mathrm{CH}$ needs only specific metrics of the constraints that is because the $\mathrm{CH}$ node acts as the aggregator that defines a specific maximization. As the number of active transmitting nodes is unknown, the parametric cannot be predicted at the initial stage.

Let $v_{c}, t_{t}$ and $t_{r}$ be the connection capacity, transmit time and receipt time of a node $n$, then use Eq. (1) to calculate the bandwidth utilization of the node wire $b_{u}$ for $m$ transmissions.

$$
\mathrm{b}_{\mathrm{u}}=\mathrm{v}_{\mathrm{c}} *\left(1-\frac{\mathrm{t}_{\mathrm{r}}-\mathrm{t}_{\mathrm{t}}}{\mathrm{t}_{\mathrm{t}}}\right), \forall \mathrm{m}
$$

The number of node-associated neighbors determines its $\operatorname{density}(\rho)$, which is estimated using Eq. (2).

$$
\rho=\left|\left\{V_{i j} \in N_{n}: \operatorname{dist}(i, j) \leq R\right\}\right|
$$

The bandwidth utilization of the node must be lower for incoming network traffic, while the density must be high for rapid switching. The above factors result in inequality in the decision of the $\mathrm{CH}$ node; therefore, the variable weight function $(\nabla w)$ is imposed on the above functions to resolve the inequality. Eqns. (3) and (4) are used to calculate the weight by acquiring the above factors.

$$
\nabla \mathrm{w}=\sum_{\mathrm{j}=1}^{\mathrm{n}} \mathrm{V}_{\mathrm{ij}} * \sigma\left(\mathrm{n}_{\mathrm{j}}\right)+\mathrm{b}_{\mathrm{u}}
$$

where,

$$
\sigma(n)=\frac{e^{\rho(n)}-e^{-\rho(n)}}{e^{\rho(n)}+e^{-\rho(n)}} \text { and } V_{i j} \in\{0,1\}
$$

The weight function is estimated to occur as a transigmoid function due to a set of observations. The higher node is considered to be the cluster header. The selected $\mathrm{CH}$ is the absolute neuron of the current observation set; the neuron is subject to change based on its effectiveness as decided to use Corollary 1. After the $\mathrm{CH}$ is selected, it sends a request for transmission to all its CMs. This request may be received by the active nodes that initiate transmission. The $\mathrm{CH}$ calculates the number of packets and checks whether the available bandwidth is sufficient to transmit them. If the available bandwidth is sufficient, the $\mathrm{CH}$ determines the number of $\mathrm{CMs}$ (including transmitting nodes) required for communication. In this case, the communication range of the $\mathrm{CH}$ is variable. The range is variable between the maximum coverage of the node and the initial $\mathrm{CH}$ range. The range cannot be reduced below the initial configuration measures for the node. The neural computing process for the selection of $\mathrm{CH}$ is shown in Figure 3.

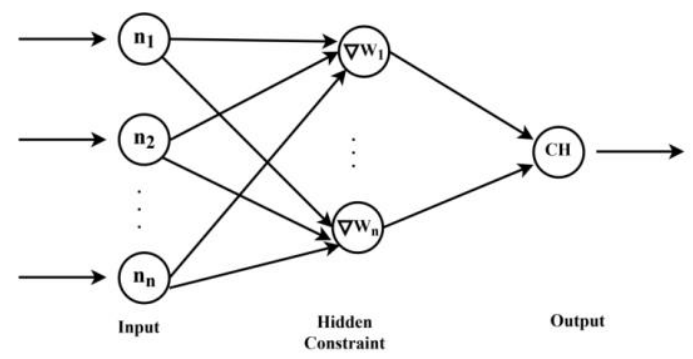

Figure 3. Neural computation process for header selection

3.3.1 Corollary 1: Considering the energy expenditure of the $\mathrm{CH}$ as its dependence factor

Analysis 1: Let us consider the problem of energy optimization as a linear bounded solution. The initial node energy $\left(E_{\text {init }}\right)$ will not remain the same if it has utilized its energy for transmitting $\left(E_{t x}\right)$ and receiving $\left(E_{\mathrm{rx}}\right)$ packets. The remaining node energy $\left(\mathrm{E}_{\mathrm{ne}}\right)$ is the dependency factor of a node. $E_{n e}=E_{\text {init }}-\left(E_{t x}+E_{r x}\right)$ needs to be estimated for the $\mathrm{CH}$ over each aggregation process.

The energy of the node cannot be regenerated for which the variable range is used as a solution. The number of nodes to be used at time $t$ is to estimate the average energy of the cluster with the current node density. The average energy of the cluster members is calculated using Eq. (5).

$$
E_{n e}(\operatorname{avg})=\sum_{i=1}^{n} \frac{E_{n e}(i)}{n}, i \neq C H
$$

If the average energy is higher than $\mathrm{CH}^{\prime} \mathrm{s} \mathrm{E}_{\mathrm{ne}}$, then the learning process is re-installed. In a re-installed learning process, the last $\mathrm{CH}$ is discarded as it does not have enough energy. Additional verification of the energy level is not necessary for this learning process. The new $\mathrm{CH}$ selected will re-center its range of communication, where $n=n-1$ will be the number of CMs before $\mathrm{R}$ variance.

The range and variance of the $\mathrm{CH}$ depend on the number of nodes involved in the communication. If the whole $\mathrm{CM}$ or the longest in-cluster $\mathrm{CM}$ is involved in the transmission process, the range does not need to be changed and therefore the connectivity at the time $(\mathrm{t}+\mathrm{x})$ remains the same.
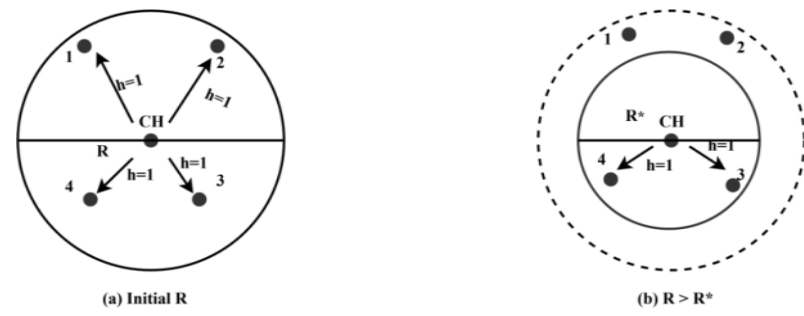

Figure 4. Range variance illustrations 
The initial value of the Connectivity $c_{n}$ is calculated using Eq. (6).

$$
c_{n}=\frac{\sum v_{i j}}{n(n-1)}
$$

If $\operatorname{dist}(\mathrm{i}, \mathrm{j})<R(\mathrm{i}) \| \mathrm{R}(\mathrm{j})$ is true then $\mathrm{v}_{\mathrm{ij}}=1$ else 0 . If only a minimum number of $\mathrm{CM}$ participates in the communication, then $\mathrm{R}$ of the $\mathrm{CH}$ is minimized to serve the incurring communicating nodes.

In that case, the new $\rho$ of the $\mathrm{CH}$ is updated and the transmitted energy is allocated for the current node density. The minimum and maximum hops up to the point where the $\mathrm{CH}$ will use energy are 1 . The nature of the estimation of the hop count in a variable $\mathrm{R}$ is shown in Figure 4.

\subsection{Learning-based routing (LR) scheme for efficient load dissemination}

As discussed in the earlier section, inadequate or defective load handling results in the network resources and output being degraded. Learning-based routing is proposed to reduce the disadvantages of data dissemination. No additional computation is required in the learning-based routing scheme; packet count, $\mathrm{p}_{\mathrm{n}}$ from the $\mathrm{CH}$ is considered to further communicate with the available neighbors. The probability of packet loss $\varnothing\left(\mathrm{p}_{\mathrm{L}}\right)$ of the forwarding, the node needs to be calculated for the transmitted position $\mathrm{p}_{\mathrm{n}}$. The number of packets and the likelihood of drop are calculated, respectively, using Eqns. (7) and (8).

$$
\mathrm{p}_{\mathrm{n}}=\frac{\mathrm{d}_{\mathrm{r}}}{\mathrm{v}_{\mathrm{t}}}
$$

where, $d_{r}$ and $v_{t}$ are the data rate and link time.

$$
\emptyset\left(\mathrm{p}_{\mathrm{L}}\right)=\mathrm{p}_{\mathrm{nmax}} *\left(\frac{\mathrm{q}_{\mathrm{a}}-\mathrm{q}_{\min }}{\mathrm{q}_{\max }-\mathrm{q}_{\min }}\right)
$$

where, $p_{n \max }$ is the maximum number of packets transmitted and $\mathrm{q}_{\mathrm{a}}, \mathrm{q}_{\min }$, and $\mathrm{q}_{\max }$ are the average, minimum, and maximum queue usage.

The learning-based routing model selects a node with a lesser drop probability that sustains maximum $\mathrm{p}_{\mathrm{n}}$.

3.4.1 Learning 1: If $\mathrm{p}_{\mathrm{n}}$ increases, $\mathrm{q}_{\mathrm{a}}$ is greater than $\mathrm{q}_{\max }$, resulting in packet loss

Solution 1: When $\mathrm{q}_{\mathrm{a}}>\mathrm{q}_{\max }$, then Equation (8) can be written as $\emptyset\left(p_{L}\right)=p_{n \max } *\left(\frac{q_{a}-q_{\min }}{q_{a}-q_{\min }}\right)=p_{\text {nmax }}$. In this case, if $\mathrm{q}_{\max }<\mathrm{p}_{\mathrm{n}}$, then overflow occurs and for all unattended transmissions, the incoming traffic is disintegrated in the same node. Therefore, the loss probability is always 1 . The learning phase is keen about minimizing loss by determining nodes that are capable of handling $\mathrm{p}_{\mathrm{n}}<\mathrm{q}_{\mathrm{a}}$. If the maximum queue size is considered, then the free space in the queue is unknown resulting in non-profit neighbor selection.

\subsubsection{Learning 2: $\mathrm{q}_{\min } \leq \mathrm{q}_{\mathrm{a}}$}

Solution 2: If the above condition is achieved, then the intermediate forwarder will have better queue size to relay information to the base station. Therefore, in this analysis, Eq. (8) can be expressed as $\emptyset\left(p_{\mathrm{L}}\right)=\mathrm{p}_{\mathrm{nmax}} *\left(\frac{\mathrm{q}_{\mathrm{a}}}{\mathrm{q}_{\max }}\right), \mathrm{q}_{\mathrm{a}} \neq \mathrm{q}_{\max }$.

The number of forwarders with this condition cannot be concurrently used, as the multipath transmission is restricted. Therefore, for ' $\mathrm{m}$ ' transmissions, a scheduling process needs to be renowned to prevent congestion free transmission. Hence, a transmission matrix is defined with the above constraints for this learning process. The transmission factor is defined with the data rate observed from the serving $\mathrm{CH}$.

The number of packets that are inconsistent with the transmission will be estimated using discriminate metric $\Delta \delta$. The data rate is mapped to the packet loss probability as an inverse function for which $\Delta \delta$ must be computed for a set of $\mathrm{m}$ transmissions. The relationship between the transmission factor and $\mathrm{q}_{\mathrm{a}}$ is represented as in Eq. (9).

$$
\left(\begin{array}{c}
\frac{1}{\mathrm{~d}_{\mathrm{r} 1}} \\
\frac{1}{\mathrm{~d}_{\mathrm{r} 2}} \\
\vdots \\
\frac{1}{\mathrm{~d}_{\mathrm{rm}}}
\end{array}\right)\left(\begin{array}{c}
\emptyset \mathrm{p}_{\mathrm{L} 1} \\
\emptyset \mathrm{p}_{\mathrm{L} 2} \\
\vdots \\
\emptyset \mathrm{p}_{\mathrm{Lm}}
\end{array}\right)=\frac{1}{\Delta \delta}\left(\begin{array}{c}
\mathrm{q}_{\mathrm{a} 1} \\
\mathrm{q}_{\mathrm{a} 2} \\
\vdots \\
\mathrm{q}_{\mathrm{am}}
\end{array}\right)
$$

Eq. (7) and Eq. (8) are used to expand Eq. (9) and are represented as Eq. (10).

$$
\left(\begin{array}{c}
\frac{1}{p_{\mathrm{n} 1} * v_{\mathrm{t} 1}} \\
\frac{1}{\mathrm{p}_{\mathrm{n} 2} * \mathrm{v}_{\mathrm{t} 2}} \\
\vdots \\
\frac{1}{\mathrm{p}_{\mathrm{nm}} * \mathrm{v}_{\mathrm{tm}}}
\end{array}\right)\left(\begin{array}{c}
\mathrm{p}_{\mathrm{n} 1} *\left(\frac{\mathrm{q}_{\mathrm{a} 1}}{\mathrm{q}_{\max 1}}\right) \\
\mathrm{p}_{\mathrm{n} 2} *\left(\frac{\mathrm{q}_{\mathrm{a} 2}}{\mathrm{q}_{\max 2}}\right) \\
\vdots \\
\mathrm{p}_{\mathrm{nn}} *\left(\frac{\mathrm{q}_{\mathrm{an}}}{\mathrm{q}_{\operatorname{maxm}}}\right)
\end{array}\right)=\frac{1}{\Delta \delta}\left(\begin{array}{c}
\mathrm{q}_{\mathrm{a} 1} \\
\mathrm{q}_{\mathrm{a} 2} \\
\vdots \\
\mathrm{q}_{\mathrm{am}}
\end{array}\right)
$$

The generalized normal form of Eq. (11) is simplified as follows:

$$
\left(\frac{1}{p_{n} * v_{t}}\right)\left(p_{n} *\left(\frac{q_{a}}{q_{\text {max }}}\right)\right)=\frac{1}{\Delta \delta}\left(q_{a}\right)
$$

The discriminate metric is computed as Eq. (12) by solving Eq. (11)

$$
\Delta \delta=\mathrm{v}_{\mathrm{t}} * \mathrm{q}_{\max }
$$

After the completion of the transmission process, the node is categorized as learning 1 or 2 . The node classified under Learning 2 is optimal and further transmission is pursued. The learning process is repeated until the next node leads to the BS. Similarly, the learning process is updated before the next communication begins.

If the node fails to learn 2, the next set of neighbors will be identified through a news broadcast. In the meantime, if the nodes $\Delta \delta$ are satisfied with the incoming network traffic, the bu adjustment concerning the neighbor density shall be made to accept the current communication set. A neighbor who does not satisfy a discriminatory metric is excluded from participating in the transmission process.

\section{PERFORMANCE ANALYSIS}

The performance of the proposed DRC-LR is evaluated through simulations using a network simulator tool.

The following metrics are estimated for assessment: Delay, 
Packet Loss, Communication Overhead, Reclustering, and Remaining Energy. Table 1 shows the parameters that are considered along with their values.

Table 1. Simulation parameters and values

\begin{tabular}{cc}
\hline Parameter & Value \\
\hline Network Region & $1000 \mathrm{~m} \mathrm{x} \mathrm{1000m}$ \\
Number of Mobile Nodes & 100 \\
MAC Protocol & 802.11 \\
Range & $250 \mathrm{~m}$ \\
Application Type & Constant Bit Rate \\
Packet Size & $512 \mathrm{~Kb}$ \\
E $_{\text {init }}$ & $16 \mathrm{~J}$ \\
Pause Time & $2 \mathrm{~ms}$ \\
Simulation Time & $300 \mathrm{~s}$ \\
Compared with & REA-MAODV [22], QUACS [23] \\
& and EE-MBOC [24, 25] \\
\hline
\end{tabular}

Notes: REA-MAODV - Real Multicast Ad hoc On-Demand Distance Vector Routing, QUACS - Queuing Ant Colony System, EE-MBOC - Energy Efficient Modified Butterfly Optimization-based Clustering.

\subsection{Delay analysis}

The comparison of DRC-LR for the delay with existing methods is shown in Figure 5. In the DRC-LR, initial communication at the node level is organized by the $\mathrm{CH}$. Cluster re-formation time is large, so no new procedures are required.

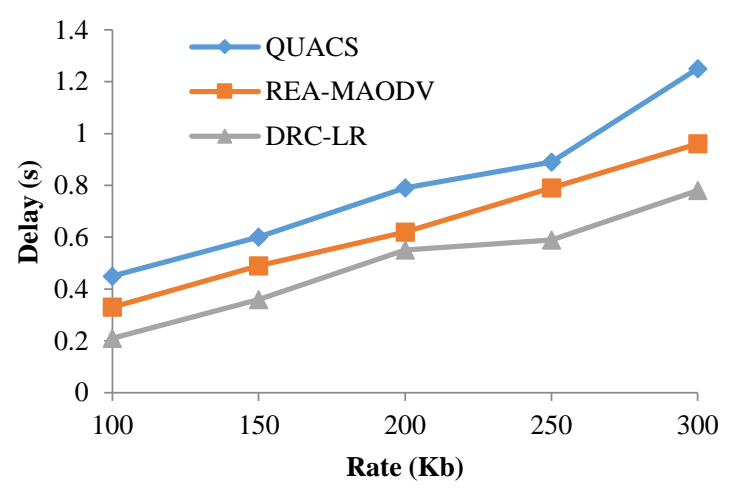

Figure 5. Delay comparison

As a result, the time complexity of cluster formation is less. The node with lower packet drops probability is selected in the LR scheme. This minimizes the number of retransmissions due to packet loss. $\mathrm{CH}$ aggregation, stable clustering, and efficient neighbor selection are the main reasons for minimizing packet loss. An increased packet rate is handled through selective neighbors to avoid delayed transmission. The proposed DRC-LR results in $37.6 \%$ and $23.20 \%$ less delay than QUACS and REA-MAODV respectively.

\subsection{Packet loss probability}

The numbers of transmissions are hidden due to the increase of packet rate and congestion. Unlike other generic methods, ratings and dissemination of data are administered through $\mathrm{q}_{\mathrm{a}}$ optimized nodes. For each transmission from the $\mathrm{CH}, \mathrm{q}_{\mathrm{a}}$ and $\mathrm{q}_{\max }$ of the node are observed.

In the learning phase, the restriction $\mathrm{q}_{\min } \leq \mathrm{q}_{\mathrm{a}}$ must be met by the node to participate in the transmission. The complete transmission process is based on the queue utilization of the nodes to the $b_{u}$ in the previous transmission. Selecting an effective $b_{u}$ node with a queue constraint improves seamless communication by reducing packet loss and it can be verified from Figure 6.

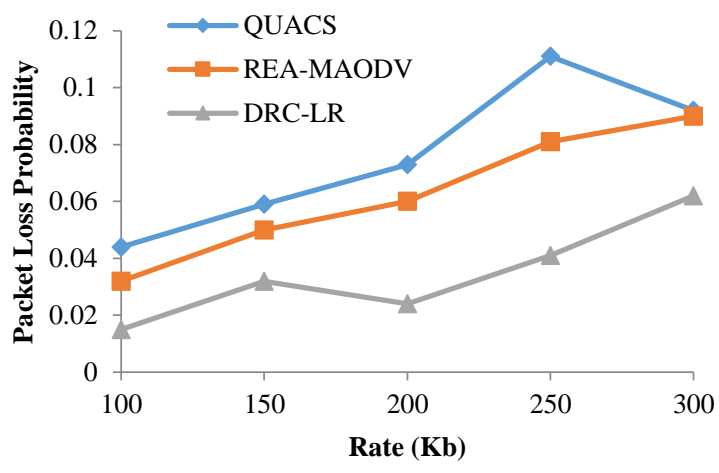

Figure 6. Packet loss probability

As a result, the probability of loss of the proposed DRCLR is $32.60 \%$ and $31.11 \%$ lower than the existing QUACS and REA-MAODV.

\subsection{Communication overhead}

The number of node replacements inside and outside the cluster is restricted using DRC and LR independently. The number of $\mathrm{CH}$-transmissions that can be changed is less within $(\mathrm{t}+\mathrm{x})$ due to $\nabla \omega$ and range variance operation. The intermediate node selection follows a queue-based verification from which a specific neighbor is selected. The need for the replacement of nodes and the discovery of new routes is, therefore lower in the proposed method. This minimizes the frequency of exchange of control messages, reducing overhead communication and it can be verified form Figure 7. DRC-LR requires $46.8 \%$ and $37.72 \%$ fewer control bits than the existing QUACS and REA-MAODV, which reduces overhead communication.

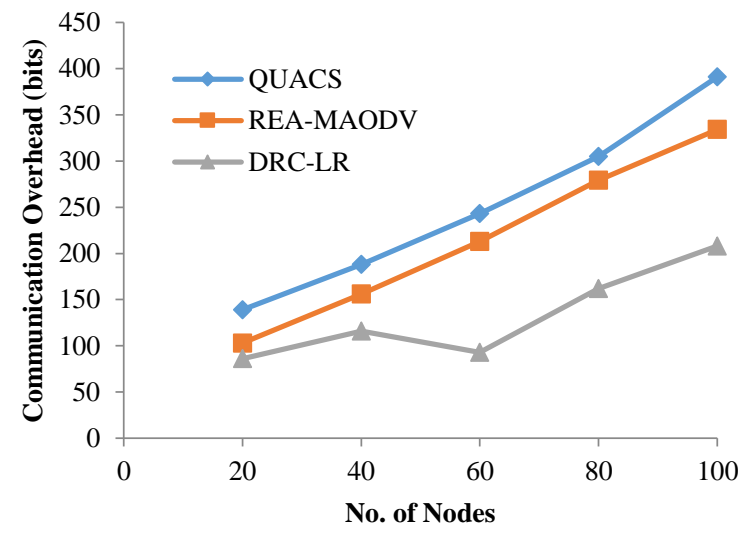

Figure 7. Communication overhead analysis

\subsection{Re-clustering instance}

In the proposed DRC-LR, cluster stability is improved by the transigmoid function. This function selects the optimal cluster header by assessing their explicit hidden constraints. Prolonged communication is ensured by using a single $\mathrm{CH}$ 
with a time $t>(t+x)$ range variance. The stability of the cluster is maintained externally by checking $\mathrm{p}_{\mathrm{L}}$ of the external nodes. From Figure 8, the need for $\mathrm{CH}$ replacement at a time $(\mathrm{t}+\mathrm{x})$ is, therefore, less than the existing EE-MBOC. Precise neighbor density factor and bandwidth utilization factors determine a reliable $\mathrm{CH}$ that sustains long-term communication. Compared to EE-MBOC, the proposed DRC-LR had a $34.17 \%$ lower chance of re-clustering.

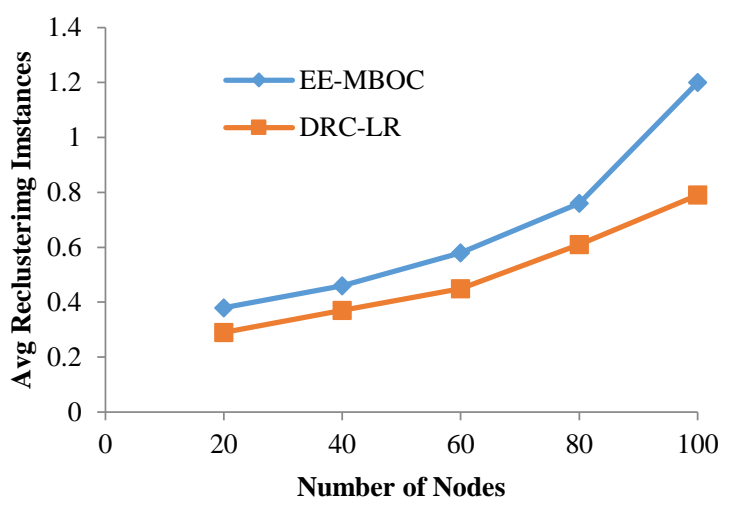

Figure 8. Re-clustering instance comparison

\subsection{Remaining energy}

Energy conservation in the proposed DRC-LR is carried out as a non-linear hidden optimization issue. In this optimization, the node maintains prolonged communication, avoiding autonomous energy expenditure.

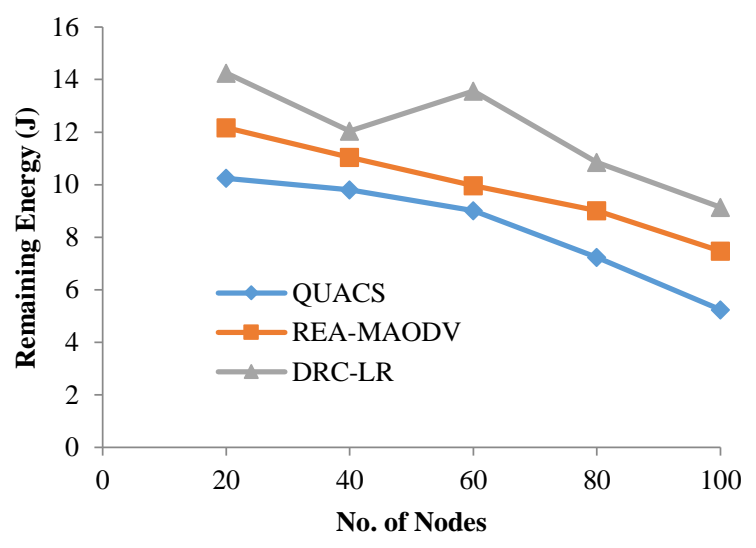

Figure 9. Remaining energy comparison

Clustering is an additional benefit in managing the network's energy consumption keeping the (n-1) nodes out; the $\mathrm{CH}$ is adjusted if its residual energy is less than the average energy. In a fixed $(t+x)$, the energy expenditure of all the nodes inside the cluster is lower. The average cluster energy retained in this method is high. The energy spent by a single $\mathrm{CM}$ is less as the nodes are least or delayed in aggregation. From Figure 9, it is confirmed that the energy stored in the network is strong. The proposed DRC-LR maintains higher remaining energy than QUACS and REAMAODV where the remaining energy is $42.66 \%$ lower and $18.27 \%$ lower than the proposed DRC-LR. Table 2 displays the outcome of the simulation executed.
Table 2. Simulation values of DRC-LR compared with QUACS and REA-MAODV

\begin{tabular}{cccc}
\hline Parameter & QUACS & $\begin{array}{c}\text { REA- } \\
\text { MAODV }\end{array}$ & $\begin{array}{c}\text { DRC- } \\
\text { LR }\end{array}$ \\
\hline Delay (s) & 1.25 & 0.96 & 0.78 \\
Packet Loss Probability & 0.092 & 0.09 & 0.062 \\
Communication & 391 & 334 & 208 \\
Overhead (bits) & 5.24 & 7.47 & 9.14 \\
Remaining Energy (J) & 5.47
\end{tabular}

\section{CONCLUSION}

In MANET load balancing, DRC-LR is proposed to increase reliability. The suggested approach operates in such a way that it enhances the efficiency of the network's data handling. The goal of DRC is to improve the stability of the cluster by selecting an enduring $\mathrm{CH}$ to aggregate and disseminate the collected data. Learning-based routing curtails the loss of packets by selecting nodes with a lower probability of loss due to increased network load. The learning process is based on the observations series over every transmission of the network. The integrated method increases network performance by reducing packet loss, delay, overhead contact, and maintaining a significant amount of node residual energy.

\section{REFERENCES}

[1] Shyam, D., Kot, A., Athalye, C. (2018). Abandoned object detection using a pixel-based finite state machine and single shot multibox detector. IEEE International Conference on Multimedia and Expo (ICME), San Diego, USA, pp. 1-6. https://doi.org/10.1109/ICME.2018.8486464

[2] Latchoumi, T.P., Jayakumar, L., Latha, P., Janakiraman, S. (2016). OFS method for selecting active features using clustering techniques. In Proceedings of the International Conference on Informatics and Analytics, ACM, Puducherry, India, pp. 1-4. https://doi.org/10.1145/2980258.2982108

[3] Li, J., Si, Y., Lang, L., Liu, L., Xu, T. (2018). A spatial pyramid pooling-based deep convolutional neural network for the classification of electrocardiogram beats. Applied Sciences, $\quad 8(9)$ : 1590. https://doi.org/10.3390/app8091590

[4] Nazila, M., Raziyeh, H., (2015). A survey on existing mechanisms in energy-aware routing in MANET, International Journal of Computer Applications Technology and Research, 4(9): 673-679. https://doi.org/10.7753/ijcatr0409.1007

[5] Ezhilarasi, T.P., Dilip, G., Latchoumi, T.P., Balamurugan, K., (2020). UIP-A smart web application to manage network environments, In Proceedings of the Third International Conference on Computational Intelligence and Informatics, pp. 97-108. https://doi.org/10.1007/978-981-15-1480-78

[6] Sundaranarayan, D., Venkatachalapathy, K. (2018). An effectual load distribution approach based on transmission power and topology controlled clustered environment in mobile Adhoc network. International Journal of Computer Applications, 179(25): 34-38. https://doi.org/10.5120/ijca2018916538 
[7] Khatoon, N., Amritanjali, (2017). Mobility aware energy efficient clustering for MANET: A bio-inspired approach with particle swarm optimization. Wireless Communications and Mobile Computing, 2017: 1-12. https://doi.org/10.1155/2017/1903190

[8] Linyang, S., Jingbo, S., Jinfeng, D. (2010). A novel energy-efficient approach to DSR based routing protocol for Ad Hoc Network. IEEE International Conference on Electrical and Control Engineering, Wuhan, China, pp. 2618-2620. https://doi.org/10.1109/iCECE.2010.1443

[9] Mandhare, V.V., Thool, R.C. (2016). Improving QoS of mobile ad-hoc network using cache update scheme in dynamic source routing protocol. Procedia Computer Science, 79: 692-699. https://doi.org/10.1016/j.procs.2016.03.090

[10] Barati, M.,Atefi, K., Khosravi, F., Daftari, Y.A. (2017). Performance Evaluation of energy efficiency for MANET using AODV routing protocol. IEEE 3rd International Conference on Computational Intelligence and Communication Technology, Kuala Lumpeu, Malaysia, pp. 636-642. https://doi.org/10.1109/ICCISci.2012.6297107

[11] Latchoumi, T.P., Balamurugan, K., Dinesh, K., Ezhilarasi, T.P. (2019). Particle swarm optimization approach for waterjet cavitation peening. Measurement, 141(6):

184-189. https://doi.org/10.1016/j.measurement.2019.04.040

[12] Mohammed, A.W., Karibasappa, K. (2012). QoS routing for heterogeneous mobile ad hoc networks, International Journal of Computer Engineering Sciences (IJCES), 2(3): 77-81.

[13] Li, G., Xu, Y. (2013). A TCP-friendly congestion control scheme for multicast with network coding. Journal of Computational Information Systems, 9(21): 8541-8548. https://doi.org/10.12733/jcis7229

[14] Chen, L., Heinzelman, W.B. (2005). QoS-aware routing based on bandwidth estimation for mobile ad hoc networks. IEEE Journal on Selected Areas in Communications, 23(3): 561-572.

[15] Chatterjee, P., Ghosh, S.C., Das, N. (2017). Load balanced coverage with graded node deployment in WSN. IEEE Transactions on Multi-Scale Computing Systems, $\quad 3(2)$ : 100-112. https://doi.org/10.1109/TMSCS.2017.2672553

[16] Delgado, O., Labeau, F., (2017). Delay-aware load balancing over multipath wireless networks. IEEE Transactions on Vehicular Technology, 66(8): 74857494. https://doi.org/10.1109/TVT.2017.2655011

[17] Aftab, F., Zhang, Z., Ahmad, A. (2017). Selforganization based clustering in MANETs using zonebased group mobility. IEEE Access, 5: 27464-27476. https://doi.org/10.1109/ACCESS.2017.2778019
[18] Selvi, F.A. (2016). Ant based multipath backbone routing for load balancing in MANET. IET Communications, $11(1)$ : 136. https://doi.org/10.1049/iet-com.2016.0574

[19] Massin, R., Martret, C.J.L., Ciblat, P. (2017). A coalition formation game for distributed node clustering in MANETs. IEEE Transactions on Wireless Communications, 16(6): 3940-3952. https://doi.org/10.1109/TWC.2017.2690419

[20] Lei, T., Wen, X., Lu, Z., Li, Y. (2017). A semimatching based load balancing scheme for dense IEEE 802.11 WLANs. IEEE Access, 5: 15332-15339. https://doi.org/10.1109/ACCESS.2017.2733083

[21] Cheng, Y., Yang, D., Zhou, H. (2018). Det-LB: A load balancing approach in 802.11 wireless networks for industrial soft real-time applications, IEEE Access, 6: 32054-32063. https://doi.org/10.1109/ACCESS.2018.2802541

[22] Liang, Q.L. (2003). Cluster head election for mobile ad hoc wireless network. 14th IEEE Proceedings on Personal, Indoor and Mobile Radio Communications, 2: 1623-1628. https://doi.org/10.1109/PIMRC.2003.1260389

[23] Cespedes-Mota, A., Castanon, G., Martínez-Herrer, A.F., Cardenas-Barron, L.E. (2018). Multi objective optimization for a wireless ad hoc sensor distribution on shaped-bounded areas. Mathematical Problems in Engineering, 2018: 1-22. https://doi.org/10.1155/2018/7873984

[24] Ahmad, M., Ikram, A.A., Wahid, I., Inam, M., Ayub, N., Ali, S. (2018). A bio-inspired clustering scheme in wireless sensor networks: BeeWSN. Procedia Computer Science, pp. https://doi.org/10.1016/j.procs.2018.04.031

[25] Hao, S., Zhang, H., Song, M. (2018). A stable and energy-efficient routing algorithm based on learning automata theory for MANET. Journal of Communications and Information Networks, 3(2): 5266. https://doi.org/10.1007/s41650-018-0012-7

\section{NOMENCLATURE}

$\begin{array}{ll}\mathrm{Kb} & \text { Kilo bytes } \\ \mathrm{ms} & \text { Microsecond }\end{array}$

\section{Greek symbols}

y Vector for all binary variables

$\omega \quad$ Trainable parameter

$\phi \quad$ Solid volume fraction

$\Theta \quad$ Dimensionless temperature

$\mu \quad$ Dynamic viscosity, $\mathrm{kg} \cdot \mathrm{m}^{-1} \cdot \mathrm{s}^{-1}$ 